\title{
Global Bifurcation of a Novel Computer Virus Propagation Model
}

\author{
Jianguo Ren, ${ }^{1}$ Yonghong $\mathrm{Xu},{ }^{2}$ and Jiming $\mathrm{Liu}^{3}$ \\ ${ }^{1}$ College of Computer Science, Jiangsu Normal University, Xuzhou 221116, China \\ ${ }^{2}$ College of Live Science, Jiangsu Normal University, Xuzhou 221116, China \\ ${ }^{3}$ Department of Computer Science, Hong Kong Baptist University, Kowloon, Hong Kong
}

Correspondence should be addressed to Jianguo Ren; rjgrjgrjgrjg@126.com

Received 7 March 2014; Accepted 27 March 2014; Published 16 April 2014

Academic Editor: Ahmed El-Sayed

Copyright (C) 2014 Jianguo Ren et al. This is an open access article distributed under the Creative Commons Attribution License, which permits unrestricted use, distribution, and reproduction in any medium, provided the original work is properly cited.

\begin{abstract}
In a recent paper by J. Ren et al. (2012), a novel computer virus propagation model under the effect of the antivirus ability in a real network is established. The analysis there only partially uncovers the dynamics behaviors of virus spread over the network in the case where around bifurcation is local. In the present paper, by mathematical analysis, it is further shown that, under appropriate parameter values, the model may undergo a global B-T bifurcation, and the curves of saddle-node bifurcation, Hopf bifurcation, and homoclinic bifurcation are obtained to illustrate the qualitative behaviors of virus propagation. On this basis, a collection of policies is recommended to prohibit the virus prevalence. To our knowledge, this is the first time the global bifurcation has been explored for the computer virus propagation. Theoretical results and corresponding suggestions may help us suppress or eliminate virus propagation in the network.
\end{abstract}

\section{Introduction}

More and more applications based on computer networks go into our daily life. While bringing convenience to us, computer networks are exposed to various threats. Computer viruses, which are programs developed to attempt to attach themselves to a host and spread to other computers mainly through Internet, can damage network resources. Consequently, understanding the law governing the spread of computer virus is of considerable interest.

Due to the high similarity between computer viruses and biological viruses [1], the classical SIR (Susceptible-InfectedRecovered) computer virus propagation model borrowed from its epidemic counterpart was proposed [2-4], which is formulated as the following system of differential equations:

$$
\begin{aligned}
& \frac{\mathrm{d} S}{\mathrm{~d} t}=b-\lambda S(t) I(t)-d S(t), \\
& \frac{\mathrm{d} I}{\mathrm{~d} t}=\lambda S(t) I(t)-\varepsilon I(t)-d I(t), \\
& \frac{\mathrm{d} R}{\mathrm{~d} t}=\varepsilon I(t)-d R(t) .
\end{aligned}
$$

Here it is assumed that all the computers connected to the network in concern are classified into three categories: susceptible, infected, and recovered computers. Let $S(t), I(t)$, and $R(t)$ denote their corresponding numbers at time $t$, respectively. This model involves four positive parameters: $b$ denotes the rate at which external computers are connected to the network, $\varepsilon$ denotes the recovery rate of infected computers due to the antivirus ability of the network, $d$ denotes the rate at which one computer is removed from the network, and $\lambda$ denotes the rate at which, when having connection to one infected computer, one susceptible computer can become infected. For some variants of this model or its extensions, see [5-19].

The use of antivirus software is regarded as one of the most effective approaches to recovering infected computers [20]. In reality, the ability of an antivirus software is usually proportional to its cost. Due to the limited software cost, the antivirus ability of a network is limited. So, it is natural to consider the following recovery function:

$$
T(I)= \begin{cases}\varepsilon I & \text { if } 0 \leq I \leq I_{0} \\ m & \text { if } I>I_{0}\end{cases}
$$


where $\varepsilon$ is the recovery rate when the antivirus ability is not fully utilized; $m=\varepsilon I_{0}$. The susceptible individuals are assumed to have the logistic growth with carrying capacity $k>0$ as well as intrinsic growth rate $r>0$.

Based on the above mentioned work, we establish a novel computer virus propagation model in [19]:

$$
\begin{aligned}
& \frac{\mathrm{d} S}{\mathrm{~d} t}=r S\left(1-\frac{S}{k}\right)-\lambda S I-d S, \\
& \frac{\mathrm{d} I}{\mathrm{~d} t}=\lambda S I-T(I)-d I, \\
& \frac{\mathrm{d} R}{\mathrm{~d} t}=T(I)-d R .
\end{aligned}
$$

Because the first two equations in (3) are independent of $R$, we can consider the following reduced model:

$$
\begin{aligned}
& \frac{\mathrm{d} S}{\mathrm{~d} t}=r S\left(1-\frac{S}{k}\right)-\lambda S I-d S, \\
& \frac{\mathrm{d} I}{\mathrm{~d} t}=\lambda S I-T(I)-d I .
\end{aligned}
$$

In [19], we carry out the global qualitative analysis of the model and obtain rich dynamical properties. First, we give the threshold value determining whether the virus dies out completely. Second, we study the existence of equilibria and investigate their local and global asymptotic stability. Next, we find that, depending on the antivirus ability, the system may undergo a local backward bifurcation or a Hopf bifurcation. Finally, we prove that, under appropriate conditions, the system may admit bistable states: a stable virus-free equilibrium and a stable virus equilibrium, or two stable virus equilibria. In this case, the initial condition is critical for the eventual steady state of the system.

In consideration of the fact that our former work on the dynamics behaviors of virus spread in model (4) is incomplete. In this paper, we continue the previous work and give detailed theoretical analysis of the presented model so as to supplement its rich dynamics. In fact, by our mathematical analysis, we have not only theoretically proved the existence of global Bogdanov-Takens bifurcation in the model, but also obtained the curves of saddle-node bifurcation, Hopf bifurcation, and homoclinic bifurcation, even for unstable limit cycle and stable virus equilibrium cases and especially found stability switches for the virus equilibria near the Bogdanov-Takens bifurcation point so that the qualitative behaviors of virus spread have been illustrated, all of which are instructive for us to choose appropriate virus-controlling strategy. On this basis, a set of policies is recommended for eradicating viruses spreading across the Internet effectively.

The remaining materials of this paper are organized this way: Section 2 studies the existence of equilibria of model (4); Section 3 examines the global Bogdanov-Takens bifurcation; Section 4 illustrates the qualitative behaviors of the bifurcation. We end the paper with a brief concluding remark in Section 5.

\section{Equilibria}

To obtain equilibria, model (4) can be written as

$$
\begin{aligned}
& r S\left(1-\frac{S}{k}\right)-d S-\lambda S I=0, \lambda S I-I(d+\varepsilon)=0, \\
& \text { if } 0 \leq I \leq I_{0}, \\
& r S\left(1-\frac{S}{k}\right)-\lambda S I-d S=0, \lambda S I-m-d I=0, \\
& \text { if } I_{0}<I .
\end{aligned}
$$

Equation (5) has a trivial equilibrium $E_{0}=(0,0)$ and a virusfree equilibrium $E_{0}^{*}=(k(r-d) / r, 0)$. Let $R_{0}=k \lambda(r-d) / r(d+$ $\varepsilon$ ) and if $R_{0}>1$, then (5) admits a unique positive solution $E^{*}\left(S^{*}, I^{*}\right)$, where

$$
S^{*}=\frac{k(r-d)}{r R_{0}}, \quad I^{*}=\frac{\left(R_{0}-1\right)(r-d)}{\lambda R_{0}} .
$$

Obviously, $E^{*}$ is a virus equilibrium of (5) if and only if

$$
1<R_{0} \leq 1+\frac{\lambda I_{0}}{\left(r-d-\lambda I_{0}\right)} .
$$

From (6) we get a quadratic equation in $I$ :

$$
k \lambda^{2} I^{2}-b I+m r=0,
$$

where $b=k \lambda(r-d)-r d$. When $b>0$ and $\Delta=b^{2}-4 k m r \lambda^{2} \geq 0$ (which is equivalent to $R_{0} \geq 1+(2 \lambda \sqrt{k m r}-r \varepsilon) / r(d+\varepsilon)$ ), (6) has the following positive solutions:

$$
I_{1}=\frac{b-\sqrt{\Delta}}{2 k \lambda^{2}}, \quad I_{2}=\frac{b+\sqrt{\Delta}}{2 k \lambda^{2}} .
$$

Accordingly,

$$
\begin{aligned}
& E_{1}=\left(S_{1}, I_{1}\right)=\left(\frac{2 k \lambda-(b-\sqrt{\Delta})}{2 \lambda r}, \frac{b-\sqrt{\Delta}}{2 k \lambda^{2}}\right), \\
& E_{2}=\left(S_{2}, I_{2}\right)=\left(\frac{2 k \lambda-(b+\sqrt{\Delta})}{2 \lambda r}, \frac{b+\sqrt{\Delta}}{2 k \lambda^{2}}\right) .
\end{aligned}
$$

$E_{1}, E_{2}$ are virus equilibria if $I_{1}, I_{2}>I_{0}$.

Let us consider the inequality $I_{1}>I_{0}$, which is equivalent to $2 k \lambda^{2} I_{0}-b \leq-\sqrt{\Delta}$. By solving it, we get

$$
1+\frac{2 k \lambda^{2} I_{0}-r \varepsilon}{r(d+\varepsilon)}<R_{0}<1+\frac{k \lambda^{2} I_{0}}{r(d+\varepsilon)} .
$$

Likewise, we can derive $I_{2}>I_{0}$ if

$$
R_{0}>1+\frac{2 k \lambda^{2} I_{0}-r \varepsilon}{r(d+\varepsilon)}
$$

or

$$
1+\frac{k \lambda^{2} I_{0}}{r(d+\varepsilon)}<R_{0}<1+\frac{2 k \lambda^{2} I_{0}-r \varepsilon}{r(d+\varepsilon)} .
$$

Notice that $R_{0}<1+k \lambda^{2} I_{0} / r(d+\varepsilon)$ is equivalent to $R_{0}<$ $1+\lambda I_{0} /\left(r-d-\lambda I_{0}\right)$. To summarize, we get the following. 
Theorem 1 (see [19]). Consider model (4). Let

$$
\begin{aligned}
& R_{0}=\frac{k \lambda(r-d)}{r(d+\varepsilon)}, \\
& p_{0}=1+\frac{2 \lambda \sqrt{k m r}-r \varepsilon}{r(d+\varepsilon)}, \\
& p_{1}=1+\frac{2 k \lambda^{2} I_{0}-r \varepsilon}{r(d+\varepsilon)}, \\
& p_{2}=1+\frac{k \lambda^{2} I_{0}}{r(d+\varepsilon)} .
\end{aligned}
$$

If $R_{0}>p_{0}$, then $E_{1}$ and $E_{2}$ are the only possible equilibria. Moreover,

(i) if $2 k \lambda^{2} I_{0}<r \varepsilon+k \lambda^{2} I_{0}$ and $p_{1}<R_{0}<p_{2}$, then both $E_{1}$ and $E_{2}$ are equilibria;

(ii) if $2 k \lambda^{2} I_{0}<r \varepsilon+k \lambda^{2} I_{0}$ and $R_{0} \geq p_{2}$, then $E_{1}$ is an equilibrium but $E_{2}$ is not;

(iii) if $2 k \lambda^{2} I_{0}>r \varepsilon+k \lambda^{2} I_{0}$, then $E_{1}$ does not exist. Furthermore, $E_{2}$ exists if and only if $R_{0}>p_{2}$.

\section{Global Bifurcation}

In this section, we will study the global bifurcation of the above model. In fact, if the condition $\Delta=b^{2}-4 k m r \lambda^{2}=0$ (which is equivalent to $R_{0}=1+(2 \lambda \sqrt{k m r}-r \varepsilon) / r(d+\varepsilon)$ ), (6) has the following unique positive solution:

$$
I_{*}=\frac{b}{2 k \lambda^{2}} .
$$

Accordingly, the virus equilibrium is

$$
E_{*}=\left(S_{*}, I_{*}\right)=\left(\frac{2 k \lambda-b}{2 \lambda r}, \frac{b}{2 k \lambda^{2}}\right) .
$$

Solving the inequality $I_{*}>I_{0}$, we can obtain

$$
R_{0}>\frac{2 k \lambda^{2} I_{0}+r d}{r(d+\varepsilon)}
$$

To summarize, we get the following.

Theorem 2. Consider model (4). Let

$$
\begin{aligned}
& R_{0}=\frac{k \lambda(r-d)}{r(d+\varepsilon)}, \\
& R_{0}^{*}=1+\frac{2 \lambda \sqrt{k m r}-r \varepsilon}{r(d+\varepsilon)}, \\
& p_{3}=\frac{2 k \lambda^{2} I_{0}+r d}{r(d+\varepsilon)}
\end{aligned}
$$

If $R_{0}^{*}=R_{0}>p_{3}$ holds, then $E_{*}$ is a unique virus equilibrium.

Theorem 3. If the virus equilibrium $E_{*}$ exists, then $E_{*}$ is a cusp of codimension 2; that is, it is a Bogdanov-Takens singularity.
Proof. To translate $E_{*}$ to the origin, let $x=S-S_{*}, y=I-I_{*}$; then (4) becomes

$$
\begin{aligned}
& \frac{\mathrm{d} x}{\mathrm{~d} t}=\left(r-\frac{2 r S_{*}}{k}-\lambda I_{*}-d\right) x-\lambda S_{*} y-\frac{r x^{2}}{k}-\lambda x y, \\
& \frac{\mathrm{d} y}{\mathrm{~d} t}=\lambda I_{*} x+\left(\lambda S_{*}-d\right) y+\lambda x y .
\end{aligned}
$$

That the Jacobin matrices at the virus equilibrium $E_{*}$ have the double-zero eigenvalue implies that

$$
\begin{gathered}
\lambda S_{*}(r-d)+\frac{2 r S_{*}\left(d-\lambda S_{*}\right)}{k}=d(r-d)-\lambda d I_{*}, \\
-\left(r-\frac{2 r S_{*}}{k}-\lambda I_{*}-d\right)=\left(\lambda S_{*}-d\right) .
\end{gathered}
$$

Let $X=x, Y=-\left(\lambda S_{*}-d\right)-y\left(\lambda S_{*}-d\right)^{2} / \lambda I_{*}$; then (21) can become the following:

$$
\begin{aligned}
& \frac{\mathrm{d} X}{\mathrm{~d} t}=Y+a_{1} X^{2}+a_{2} X Y, \\
& \frac{\mathrm{d} Y}{\mathrm{~d} t}=b_{1} X^{2}+b_{2} X Y,
\end{aligned}
$$

where $a_{1}=\left(\left(\lambda S_{*}-d\right) / S_{*}-r / k\right), a_{2}=1 / S_{*}, b_{1}=\left(\lambda S_{*}-d\right)(\lambda-$ $\left.a_{1}\right)$, and $b_{2}=\lambda-a_{2}\left(\lambda S_{*}-d\right)$.

Again by letting $x=X-(1 / 2) a_{2} X^{2}, y=Y+a_{1} X^{2}$, we can obtain the following:

$$
\begin{gathered}
\frac{\mathrm{d} x}{\mathrm{~d} t}=y+R_{1}(x, y), \\
\frac{\mathrm{d} y}{\mathrm{~d} t}=b_{1} x^{2}+\left(b_{2}+2 a_{1}\right) x y+R_{2}(x, y),
\end{gathered}
$$

where $R_{1}, R_{2}$ are smooth functions in $(x, y)$ at least of third order and $b_{1}=\left(\lambda S_{*}-d\right)\left(\lambda-a_{1}\right)=(b / 2 r)\left(d / S_{*}+r / k\right)>$ $0, b_{2}=\lambda-a_{2}\left(\lambda S_{*}-d\right)=d / S_{*}>0$. It follows from [21, 22] that model (4) admits a Bogdanov-Takens bifurcation.

Next, we will explore the approximating BT bifurcation curves by choosing $m$ and $r$ as bifurcation parameters. First we fix the parameters $k=k_{0}, \lambda=\lambda_{0}$, and $d=d_{0}$ and let $r=r_{0}+\lambda_{1}, m=m_{0}+\lambda_{2}$, where $\lambda_{1}$ and $\lambda_{2}$ are parameters which vary in a small neighborhood of the origin. Let $x=$ $S-S_{*}, y=I-I_{*}$; then $E_{*}$ is translated to $(0,0)$ and model $(4)$ becomes

$$
\begin{aligned}
& \frac{\mathrm{d} x}{\mathrm{~d} t}=a_{1} \lambda_{1}+a_{2} x-a_{3} y-a_{4} x^{2}-\lambda x y, \\
& \frac{\mathrm{d} y}{\mathrm{~d} t}=-\lambda_{2}+\lambda I_{*} x+\left(\lambda S_{*}-d\right) y+\lambda x y,
\end{aligned}
$$

where $a_{1}=\lambda_{1} S_{*}\left(1-S_{*} / k\right), a_{2}=r_{0}+\lambda_{1}+2 S_{*}\left(r_{0}-\lambda_{1}\right) / k-$ $\lambda I_{*}-d, a_{3}=\lambda S_{*}$, and $a_{4}=\left(r_{0}+\lambda_{1}\right) / k$. 
Let $X=x, Y=a_{1} \lambda_{1}+a_{2} x-a_{3} y-a_{4} x^{2}-\lambda x y$; then (24) becomes

$$
\begin{gathered}
\frac{\mathrm{d} X}{\mathrm{~d} t}=Y, \\
\frac{\mathrm{d} y}{\mathrm{~d} t}=b_{1}+b_{2} X+b_{3} Y+b_{4} X^{2} \\
+b_{5} X Y+b_{6} Y^{2}+R_{3}(X, Y),
\end{gathered}
$$

where

$$
\begin{aligned}
& b_{1}=-\lambda_{2} a_{3}-a_{1}\left(\lambda_{0} S_{*}-d\right), \\
& b_{2}=-\lambda_{2} \lambda_{0}-a_{3} \lambda_{0} I_{*}-a_{2}\left(\lambda_{0} S_{*}-d\right)-a_{1} \lambda_{0} \\
& b_{3}=a_{2}-\frac{\lambda_{0}\left(a_{1}+1\right)}{a_{3}}+\left(\lambda_{0} S_{*}-d\right), \\
& b_{4}=-\lambda_{0}^{2} I_{*}+a_{4}\left(\lambda_{0} S_{*}-d\right)-a_{2} \lambda_{0} \\
& b_{5}=\lambda_{0}-2 a_{4}-\lambda_{0} \frac{a_{2}}{a_{3}}+\lambda_{0}^{2} \frac{a_{1}}{a_{3}^{2}} \\
& b_{6}=\frac{\lambda_{0}}{a_{3}}
\end{aligned}
$$

and $R_{3}(X, Y)$ is a smooth function of $X, Y$ at least of third order.

Let $x=X+b_{3} / b_{5}, y=Y$; then (26) becomes

$$
\begin{gathered}
\frac{d x}{d t}=y \\
\frac{\mathrm{d} y}{\mathrm{~d} t}=h_{1}+h_{2} x+b_{4} x^{2}+b_{5} x y+b_{6} y^{2}+R_{4}(x, y),
\end{gathered}
$$

where $h_{1}=b_{1}+b_{4} b_{3}^{2} / b_{5}^{2}-b_{2} b_{3} / b_{5}, h_{2}=b_{2}-2 b_{3} b_{4} / b_{5}$, and $R_{4}(x, y)$ is a smooth function of $x, y$ at least of third order.

A new time variable $\tau$ is introduced which satisfies $\mathrm{d} t=$ $\left(1-b_{6} x\right) \mathrm{d} \tau$. Still write $\tau$ as $t$; then (27) can be written as follows:

$$
\begin{gathered}
\frac{\mathrm{d} x}{\mathrm{~d} t}=\left(1-b_{6} x\right) y, \\
\frac{\mathrm{d} y}{\mathrm{~d} t}=\left(1-b_{6} x\right)\left(h_{1}+h_{2} x+b_{4} x^{2}+b_{5} x y+b_{6} y^{2}\right) \\
+R_{5}(x, y),
\end{gathered}
$$

where $R_{5}(x, y)$ is a smooth function of $x, y$ at least of third order.

Let $X=x, Y=\left(1-b_{6} x\right) y$, rewrite $X, Y$ as $x, y$, and then (28) becomes

$$
\begin{gathered}
\frac{\mathrm{d} x}{\mathrm{~d} t}=y, \\
\frac{\mathrm{d} y}{\mathrm{~d} t}=c_{1}+c_{2} x+c_{3} x^{2}+c_{4} x y+R_{6}(x, y),
\end{gathered}
$$

where $c_{1}=h_{1}, c_{2}=h_{2}-2 h_{1} b_{6}, c_{3}=b_{4}+h_{1} b_{6}^{2}$, and $c_{4}=$ $b_{5}, R_{6}(x, y)$ is a smooth function of $x, y$ at least of third order.

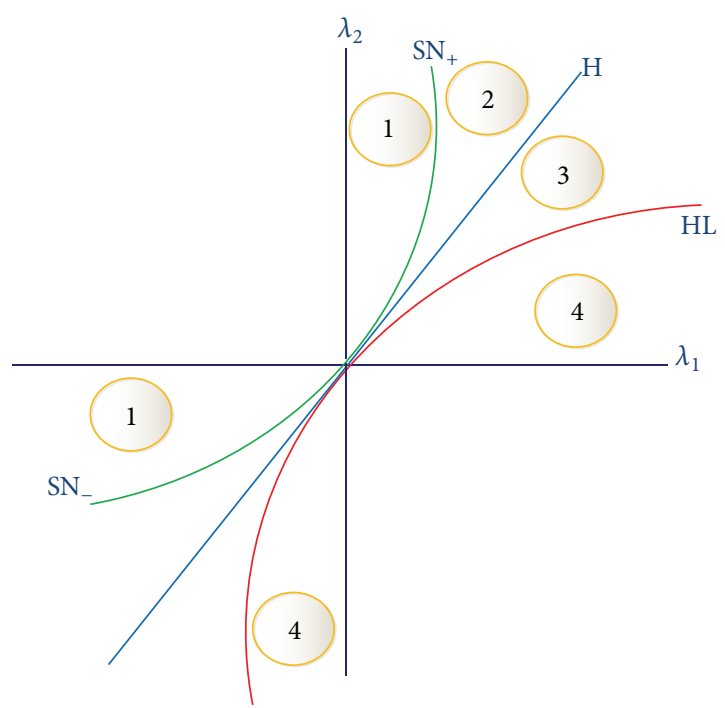

Figure 1: The bifurcation diagram of our model.

Let $X=\left(c_{4}^{2} / c_{3}\right) x, Y=\left(c_{4}^{3} / c_{3}^{2}\right) y$, and $\tau=\left(c_{3} / c_{4}\right) t$, rewrite $X, Y, \tau$ as $x, y, t$, and then (29) can become the following:

$$
\begin{gathered}
\frac{\mathrm{d} x}{\mathrm{~d} t}=y, \\
\frac{\mathrm{d} y}{\mathrm{~d} t}=\beta_{1}+\beta_{2} x+x^{2}+x y+R_{7}(x, y),
\end{gathered}
$$

where $\beta_{1}=c_{1} c_{4}^{4} / c_{3}^{3}, \beta_{2}=c_{2} c_{4}^{2} / c_{3}^{2}$, and $R_{7}(x, y)$ is a smooth function of $x, y$ at least of third order.

According to the theorems of [23], we can obtain the following theorem:

Theorem 4. If the virus equilibrium $E_{*}$ exists, then model (4) admits the following bifurcation behavior:

(1) there is a saddle-node bifurcation curve $S N=\left\{\left(\lambda_{1}, \lambda_{2}\right)\right.$ : $\left.4 \beta_{1}=\beta_{2}^{2}+o\left(\|\lambda\|^{2}\right)\right\}$

(2) there is a Hopf bifurcation curve $H=\left\{\left(\lambda_{1}, \lambda_{2}\right): \beta_{1}+\right.$ $\left.o\left(\left\|\lambda_{1}, \lambda_{2}\right\|^{2}\right)=0, \beta_{2}<0\right\} ;$

(3) there is a homoclinic curve $H L=\left\{\left(\lambda_{1}, \lambda_{2}\right): 25 \beta_{1}+\right.$ $\left.6 \beta_{2}^{2}=0+o\left(\left\|\lambda_{1}\right\|^{2}\right)\right\}$.

\section{Further Discussions and Suggestions}

From the above theorem, we have found that, depending on the parameters $\left(\lambda_{1}, \lambda_{2}\right)$ that vary in a small neighborhood of the original, model (4) undergoes a B-T bifurcation consisting of the curves of saddle-node bifurcation, Hopf bifurcation, and homoclinic bifurcation where the virus exhibits the different spread behaviors. In the following, we will illustrate the qualitative behaviors and the bifurcation diagram as sketched in Figure 1.

Now, Let us make a roundtrip near the Bogdanov-Takens point $\lambda=0$. When the point lies in region 1 where there are no virus equilibria (and thus no limit cycles are possible) and the 
corresponding flow is parallelizable. When the point lies on the curve $\mathrm{SN}_{+}$, model (4) has exactly one virus equilibrium $E$, which is saddle-node, and $E$ has three separatrices: one of them tends to $E$ as $t$ to $-\infty$ (unstable separatrix) and two are stable separatrices (tend to $E$ as $t$ to $+\infty$ ). Entering from region 2 into region 3 through the component $\mathrm{SN}_{+}$of the fold curve yields two virus equilibria: a saddle and a stable node and has no periodic solution. It is worth noting that when the points pass from one side of the cure $\mathrm{SN}_{+}$to the other side, the number of equilibria of model (4) changes from zero to two. This hints that model (4) undergoes a saddle-node bifurcation of codimension 1, and the surface $\mathrm{SN}$ is called a saddle-node bifurcation surface. Then, the node turns into a focus and loses stability when crossing the Hopf bifurcation boundary $H$. While entering into region 3 , there exist exactly two virus equilibria: one saddle point and another stable focus surrounded by one limit cycle, which is unstable. As we move clockwise, it "grows" and approaches the saddle, turning into a homoclinic orbit at HL. For our remainder journey clockwise, no limit cycles must remain. Therefore, there must be global bifurcations "destroying" the cycle somewhere between $H$ and $\mathrm{SN}_{-}$. When reaching curve $\mathrm{HL}$, two virus equilibria appear, one saddle point and one stable focus. Two separatrices of the saddle point form a closed separatrix loop surrounding the stable focus. There is no periodic solution and if we trace the homoclinic orbit along the curve HL toward the Bogdanov-Takens point, the looplike orbit shrinks and disappears. To complete our roundtrip, note that there are no cycles in region 4 located below the curve HL, and a stable node and a saddle, existing for the parameter values in these regions, collide and disappear, consequently, resulting in only one virus equilibrium which has three separatrices, one unstable and two stable at the fold curve $\mathrm{SN}_{-}$. Let us also point out that at $\lambda=0$ the critical equilibrium with a double-zero eigen value has exactly two asymptotic orbits (one tending to the equilibrium for $t \rightarrow$ $+\infty$ and one approaching it as $t \rightarrow-\infty$ ).

We know that the virus propagation behaviors mainly depend on the dynamics, specifically, the existence and stability of virus-free equilibrium or virus equilibrium and the limit cycles of model. Based on the above analysis, we can see that the dynamics of B-T bifurcation can broadly fall into three types between different regions and curves: unstable virus equilibrium, no virus equilibrium, and no limit cycle; stable virus equilibrium; stable virus equilibrium and unstable limit cycle. Next, we provide some suggestions aiming at each type to suppress or eliminate the virus spread in the real network.

Case 1. Existence of an unstable virus equilibrium, no equilibrium, and no limit cycle: this implies that any positive orbit of model (4) meets the positive $S$-axis in finite time; that is, there is no infected computer in the network, and therefore, the virus will become extinct. Thus, the virus should be left alone in a network.

Case 2. Existence of a stable virus equilibrium: this implies that the virus cannot be eradicated, but can be suppressed.

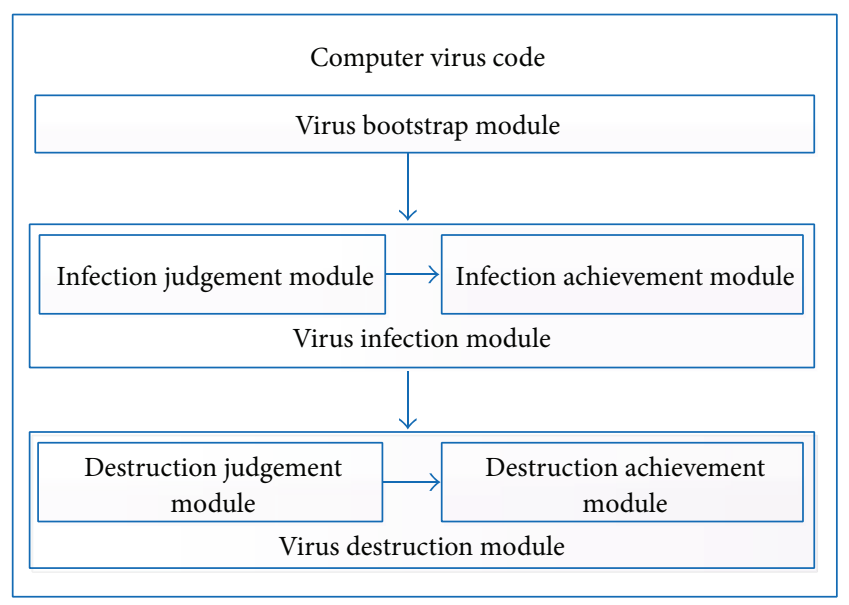

FIgURE 2: The logic structure of virus.

Thus, virus prevalence, which is defined as the infected computers, independent of any initial state, would attain a certain level. So, it is necessary for us to carry out certain steps to inhibit the virus prevalence to an acceptable level. A proper suggestion is to increase $\lambda_{2}$, which corresponds to the increase of the antivirus ability $m$. Say, it is sufficient to increase $m$ to the extent where a virus equilibrium becomes unstable so as to eliminate the virus in a real network. This leads us to perceive that, on the one hand, we should understand the structure of currently prevalent viruses, because the new antivirus software developed based on this understanding would possess a powerful capability to detect and clear existing viruses; toward this direction, we give the structure of the virus in Figure 2. On the other hand, we should invest more on developing antivirus software or updating it timely so as to enhance its antivirus ability.

Case 3. Coexistence of a stable virus equilibrium and an unstable limit cycle, that is, a unique unstable limit cycle inside which the positive orbits of model (4) tend to a virus equilibrium as $t$ tends to infinity: this implies that virus is persistent inside the cycle, whereas the virus becomes disappeared outside this cycle, which leads us to perceive that, on the one hand, we should enhance the antivirus ability of the network; on the other hand, we should take proper measures to make the initial position lie outside the cycle so as to eliminate the virus.

\section{Concluding Remarks}

In our previous work, we presented a novel computer virus model describing the virus spread behaviors under the effect of the antivirus ability in a real network and analyzed its dynamics for the case where around bifurcation is local. In order to supplement these partial dynamical behaviors, in the present paper, it is further shown that, under appropriate parameter values, the model may undergo a global B-T bifurcation, and the curves of saddle-node bifurcation, Hopf bifurcation, and homoclinic bifurcation are obtained at the virus equilibrium. To our knowledge, this is the first time the 
global bifurcation has been explored for the computer virus propagation. Based on the theoretical results, corresponding suggestions may help us take practical measures to suppress or eliminate virus in the network.

Towards this direction, there are some further issues that are yet to be studied. First, the inherent structure of a computer virus represents the resource of its breakout. Therefore, it is crucial to dissect the logical structure of virus and analyze the function of each of the modules and their interrelations so as to uncover the resource of virus burst. Second, it is well known that a computer virus may spread through the Internet, which can be reflected in two-layer structure: router layer and autonomous systems layer. The former processes different extents of the antivirus ability due to the limit cost of antivirus and the latter emerges powerlaw distribution topology features [24], both of which will be combined to model and analyze the virus propagation behaviors on the Internet in our next work.

\section{Conflict of Interests}

The authors declare that there is no conflict of interests regarding the publication of this paper.

\section{Acknowledgments}

The work is supported by the National Natural Science Foundation of China under Grant no. 61304117, the Natural Science Foundation of the Jiangsu Higher Education Institutions of China under Grant no. 13KJB520008, and the doctorate teacher support project of Jiangsu Normal University under Grant no. 12XLR021.

\section{References}

[1] F. Cohen, "Computer viruses. Theory and experiments," Computers \& Security, vol. 6, no. 1, pp. 22-35, 1987.

[2] J. O. Kephart, T. Hogg, and B. A. Huberman, "Dynamics of computational ecosystems," Physical Review A, vol. 40, no. 1, pp. 404-421, 1989.

[3] J. C. Wierman and D. J. Marchette, "Modeling computer virus prevalence with a susceptible-infected-susceptible model with reintroduction," Computational Statistics \& Data Analysis, vol. 45, no. 1, pp. 3-23, 2004.

[4] J. R. C. Piqueira and V. O. Araujo, "A modified epidemiological model for computer viruses," Applied Mathematics and Computation, vol. 213, no. 2, pp. 355-360, 2009.

[5] C. Gan, X. Yang, W. Liu, Q. Zhu, and X. Zhang, "An epidemic model of computer viruses with vaccination and generalized nonlinear incidence rate," Applied Mathematics and Computation, vol. 222, pp. 265-274, 2013.

[6] B. K. Mishra and N. Jha, "Fixed period of temporary immunity after run of anti-malicious software on computer nodes," Applied Mathematics and Computation, vol. 190, no. 2, pp. 12071212, 2007.

[7] B. K. Mishra and N. Jha, "SEIQRS model for the transmission of malicious objects in computer network," Applied Mathematical Modelling, vol. 34, no. 3, pp. 710-715, 2010.

[8] L.-P. Song, Z. Jin, G.-Q. Sun, J. Zhang, and X. Han, "Influence of removable devices on computer worms: dynamic analysis and control strategies," Computers \& Mathematics with Applications, vol. 61, no. 7, pp. 1823-1829, 2011.

[9] C. Gan, X. Yang, W. Liu, and Q. Zhu, "A propagation model of computer virus with nonlinear vaccination probability," Communications in Nonlinear Science and Numerical Simulation, vol. 19, no. 1, pp. 92-100, 2014.

[10] C. Zhang, Y. Zhao, and Y. Wu, "An impulse model for computer viruses," Disrete Dynamics in Nature and Society, vol. 2012, Article ID 260962, 13 pages, 2012.

[11] C. Zhang, Y. Zhao, Y. Wu, and S. Deng, "A stochastic dynamic model of computer viruses," Discrete Dynamics in Nature and Society, vol. 2012, Article ID 264874, 16 pages, 2012.

[12] J. B. Shukla, G. Singh, P. Shukla, and A. Tripathi, "Modeling and analysis of the effects of antivirus software on an infected computer network," Applied Mathematics and Computation, vol. 227, pp. 11-18, 2014.

[13] Q. Zhu, X. Yang, L.-X. Yang, and X. Zhang, "A mixing propagation model of computer viruses and countermeasures," Nonlinear Dynamics, vol. 73, no. 3, pp. 1433-1441, 2013.

[14] C. Gan, X. Yang, Q. Zhu, J. Jin, and L. He, "The spread of computer virus under the effect of external computers," Nonlinear Dynamics, vol. 73, no. 3, pp. 1615-1620, 2013.

[15] K. M. Fuhrman, G. A. Pinter, and J. A. Berges, "Dynamics of a virus-host model with an intrinsic quota," Mathematical and Computer Modelling, vol. 53, no. 5-6, pp. 716-730, 2011.

[16] J. Amador, "The stochastic SIRA model for computer viruses," Applied Mathematics and Computation, vol. 232, pp. 1112-1124, 2014.

[17] L.-X. Yang and X. Yang, "The spread of computer viruses under the influence of removable storage devices," Applied Mathematics and Computation, vol. 219, no. 8, pp. 3914-3922, 2012.

[18] F. Wang, Y. Zhang, C. Wang, J. Ma, and S. Moon, "Stability analysis of a SEIQV epidemic model for rapid spreading worms," Computers \& Security, vol. 29, no. 4, pp. 410-418, 2010.

[19] J. Ren, X. Yang, Q. Zhu, L.-X. Yang, and C. Zhang, "A novel computer virus model and its dynamics," Nonlinear Analysis: Real World Applications, vol. 13, no. 1, pp. 376-384, 2012.

[20] S. Forrest, S. A. Hofmeyr, and A. Somayaji, "Computer Immunology," Communications of the ACM, vol. 40, no. 10, pp. 88-96, 1997.

[21] R. Bogdanov, "Bifurcation of a limit cycle for a family of vector fields on the plane," Selecta Mathematica Soviet, vol. 1, pp. 373388, 1981.

[22] R. Bogdanov, "Versal deformations of a singular point on the plane in the case of zero eigenvalues," Selecta Mathematica Soviet, vol. 1, pp. 389-421, 1981.

[23] J. E. Marsden and L. Sirovich, Applied Mathematical Sciences, Springer, New York, NY, USA, 1998.

[24] L. A. Adamic and B. A. Huberman, "Power-law distributionof the world wide web," Science, vol. 287, no. 5461, p. 2115, 2000. 


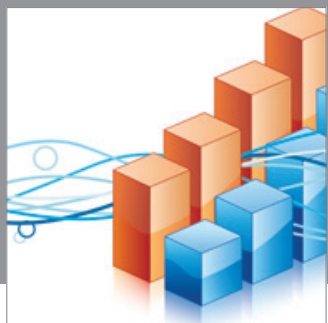

Advances in

Operations Research

mansans

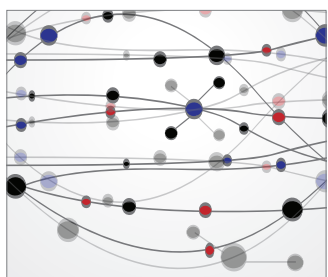

The Scientific World Journal
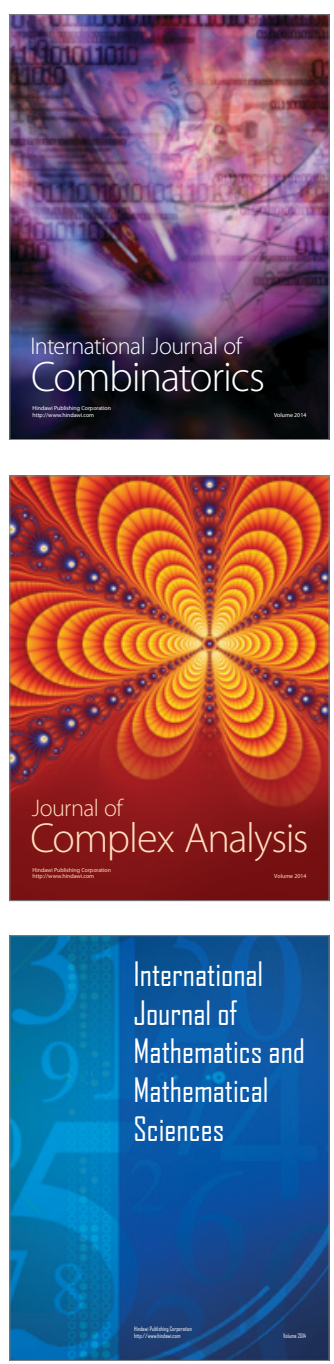
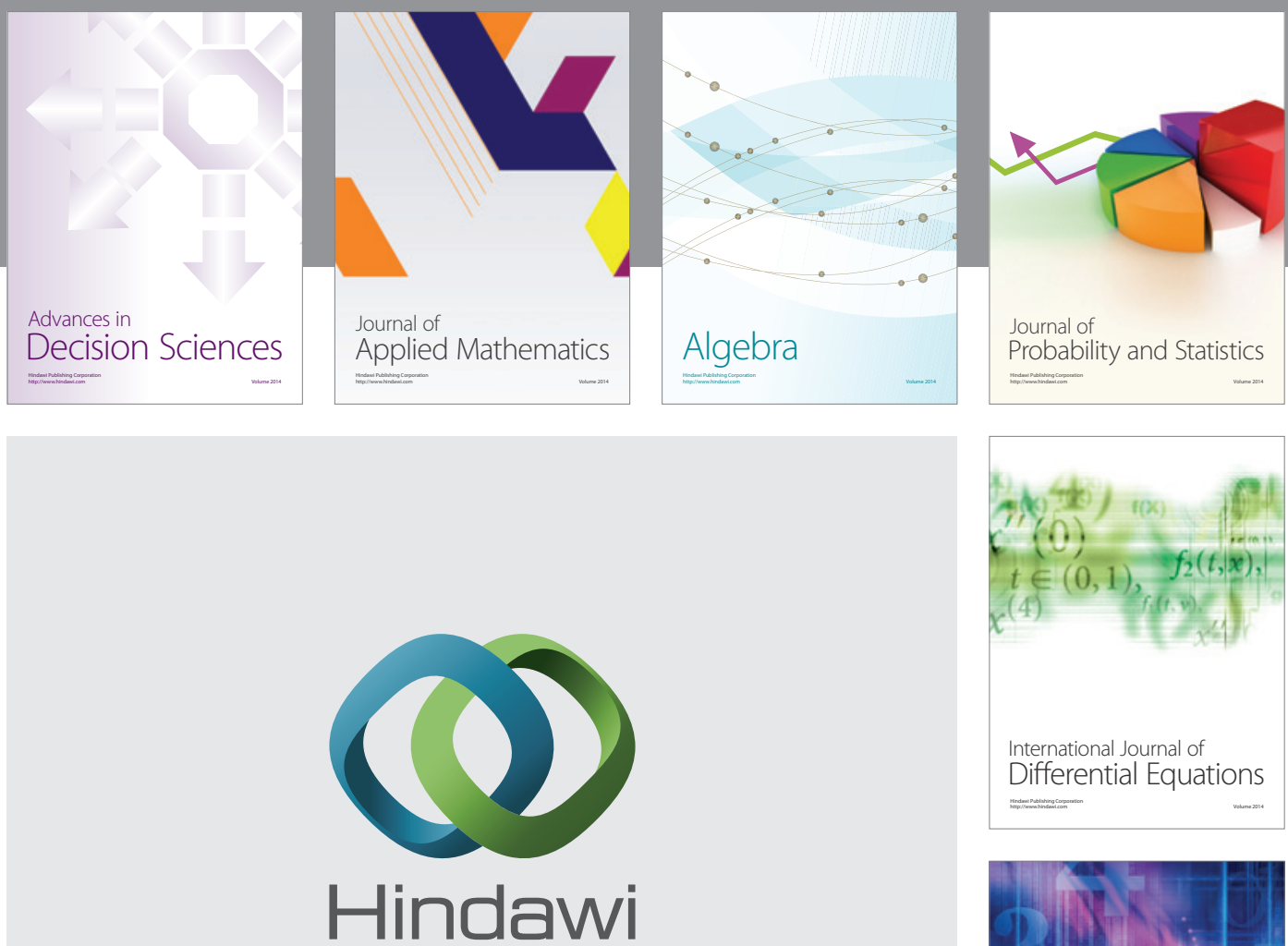

Submit your manuscripts at http://www.hindawi.com
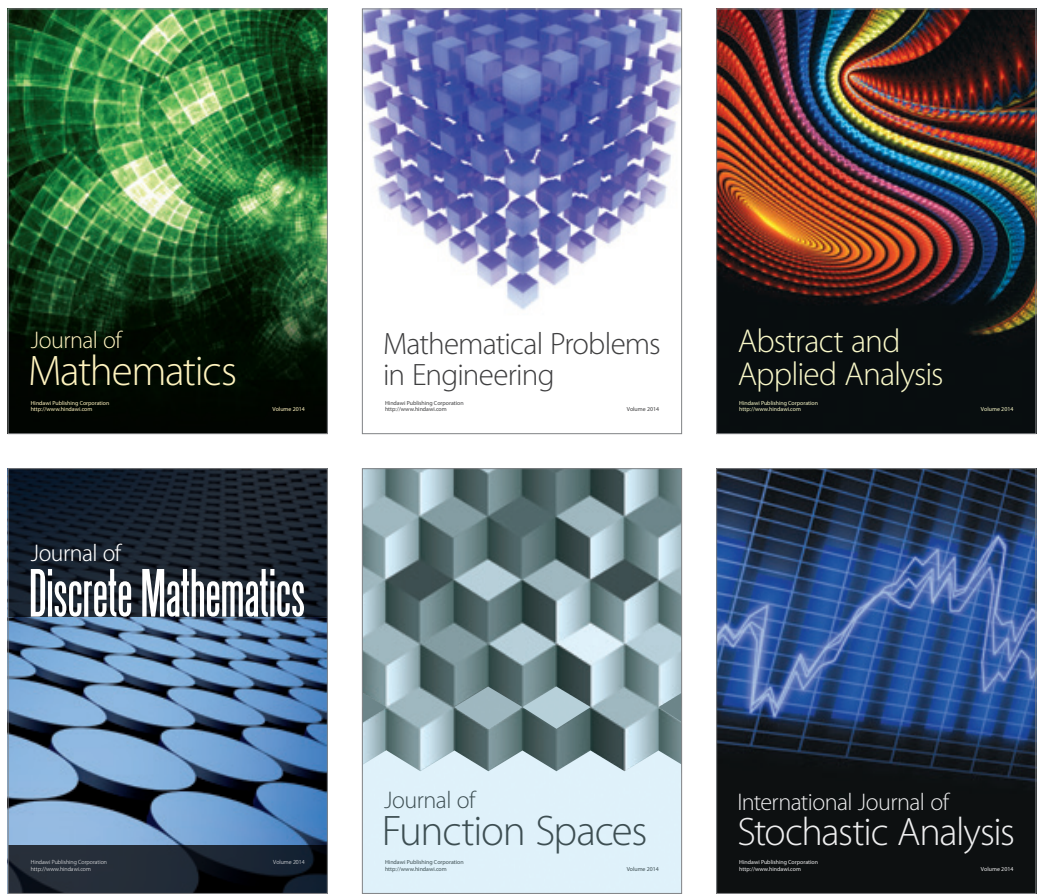

Journal of

Function Spaces

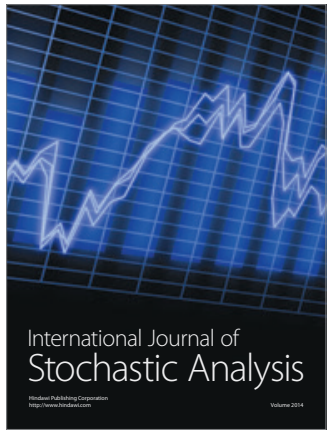

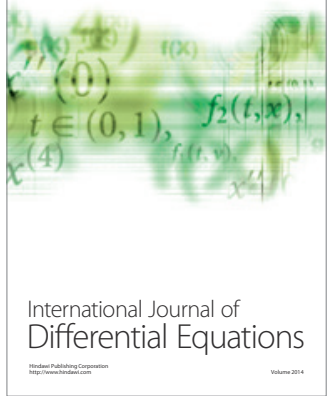
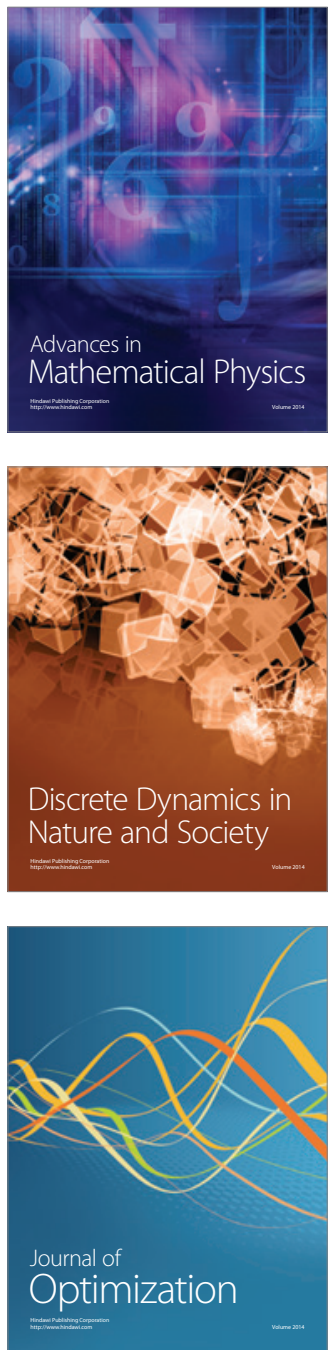